\title{
Representaciones Sociales de Profesores y Estudiantes sobre las Personas LGBTI: Un Estudio de Caso en un Liceo Católico y Laico
}

\section{Social Representations of Teachers and Students about LGBTI People: A Case Study in a Catholic and Secular Secondary School}

\author{
Evelyn Toro Villagra ${ }^{1 *}$ \\ Pablo Moya Rojas ${ }^{2}$ \\ Rolando Poblete Melis ${ }^{3}$ \\ ${ }^{1}$ Colegio Inmaculada Concepción, Chile \\ ${ }^{2}$ Colegio Los Pensamientos, Chile \\ ${ }^{3}$ Universidad Central de Chile, Chile
}

\begin{abstract}
Hoy nos enfrentamos a una revitalización de la discusión respecto de la diversidad sexual en el contexto educativo, sin embargo, no se puede desconocer que las orientaciones que cada establecimiento tenga respecto de la concepción de diversidad sexual pueden marcar diferencias significativas en torno a las ideas y actuaciones que llevan a cabo en sus comunidades. En ese marco, la investigación que da origen a este artículo se propuso identificar las representaciones sociales que tienen docentes y estudiantes acerca de la inclusión de las temáticas y estudiantes LGTBI en un centro educativo secundario de orientación católica y otro laico de la ciudad de Santiago. A través de la aplicación de entrevistas en profundidad a docentes y estudiantes, se pudo establecer que existen diferencias relevantes en ambos establecimientos respecto de la forma de abordar la diversidad sexual, su visibilidad y las creencias que los actores tienen al respecto.
\end{abstract}

Descriptores: Educación Integradora; Discriminación sexual; Estereotipo sexual; Derechos humanos; Estudiante.

Nowadays we are facing a revitalization of the discussion regarding sexual diversity in the educational context. However, we cannot ignore that the orientations that each establishment has regarding sexual diversity can lead to significant differences when it comes to the beliefs and actions that they carry out in their communities. The research that gives rise to this article aimed at identifying the social representations of teachers and students regarding the inclusion of LGTBI subjects and students in a Catholic secondary school and another secular one in the city of Santiago. Through the application of in-depth interviews with teachers and students, it was established that there are relevant differences between these establishments regarding the way to approach sexual diversity, its visibility and the beliefs that the actors have in this regard.

Keywords: Inclusive education; Sexual discrimination; Sexual stereotype; Human rights; Students. 


\section{Introducción}

El año 2014 en Chile, la psicóloga Leslie Nicholls, en conjunto con Ramón Gómez y el patrocinio de la Junta Nacional de Jardines Infantiles (JUNJI) y otras instituciones, lanzó el libro "Nicolás tiene 2 papás", texto que expone la cotidianeidad de un niño hijo de una pareja homoparental. El libro fue repartido de manera gratuita en bibliotecas y centros educativos. A partir de esto comenzó una discusión a nivel social, político y religioso en cuanto a las implicancias de este cuento y el mensaje que transmitía a niños y niñas (Núñez, 2014).

Una situación similar ocurrió en cuanto al revuelo provocado durante julio del 2017 cuando recorrió las calles de Santiago el "bus de la libertad", auspiciado por la organización Citizengo que buscaba defender la vida, la familia y la libertad, en conjunto con la agrupación de "Padres Objetores de Chile" y el observatorio legislativo cristiano. Ante esta situación, y como forma de protesta, en simultáneo circuló por las calles de la capital el "bus de la diversidad" promovido por organizaciones como el Movimiento de Liberación Homosexual (Monzón, 2017).

Estas situaciones y el creciente debate en torno a estos temas dan cuenta de la mayor visibilidad que ha alcanzado en los últimos años la diversidad sexual y de género en nuestro país, siendo el propio Estado también un actor relevante. En efecto, a través de políticas educativas (Programas de Educación Sexual; Orientaciones respecto de la incorporación de niños y niñas y trans en el ámbito educativo, Ley de Inclusión), se ha ido posicionando un discurso que se mueve entre la lógica antidiscriminación y en menor medida aquella que establece derechos sociales para Lesbianas, Gay, Bisexuales, Trans e Inter (en adelante LGTBI) (Galaz, Sepúlveda, Poblete, Troncoso y Morrison, 2018), superando las visiones tradicionales reducidas a un abordaje exclusivamente médicojurídico (González, Núñez, Galaz, Troncoso y Morrison, 2018).

De esta forma, y cada vez con mayor frecuencia las personas conversan y debaten acerca de diversidad sexual, pero paradójicamente en las escuelas la situación es diferente, toda vez que cuando se habla de diversidad sexual y estudiantes LGTBI es a causa de la discriminación o los déficits que posee en relación al tema esta institución (Astudillo, 2016; Cornejo, 2018; Galaz et al., 2018; Movilh, 2008; UNESCO, 2015; Torres, 2013). Como dato, el $61 \%$ de los estudiantes en Chile acusa haber sido testigo de bullying homofóbico por parte de los profesores y un $77 \%$ reconoce nunca haber conversado del tema en clases de orientación (Barbero y Pichardo, 2016; Rehbein, 2017).

Pareciera que los establecimientos educacionales de nuestro país invisibilizan todo lo que tenga que ver con la comunidad LGBTI de manera institucional. En esa misma línea, Cornejo (2014) señala que, aunque la homofobia se ha manifestado de diversas formas en el sistema educativo, su invisibilización ha sido recurrente, desconociéndose sus inclinaciones erótico-afectivas, lo que ha derivado en una heterosexualidad impuesta en especial en el currículo.

La educación sexual -como mecanismo que puede aportar en la línea de promover el respeto de la diversidad sexual- no ha sido "explícitamente obligatoria dentro del ordenamiento jurídico chileno, quedando la implementación de los respectivos programas a merced de la libertad de enseñanza de cada escuela, tal como lo dispuso la Ley Orgánica Constitucional de Educación" (Arenas et al., 2016, p. 17). A lo anterior se suma el hecho que al menos desde la década del sesenta, cuando se inician los primeros programas de 
educación sexual, y hasta la actualidad, la tendencia es a la invisibilización por ejemplo del homoerotismo (Cornejo, 2014), legitimando una visión heternormativa de la sexualidad. Por otro lado, la perspectiva que ha primado respecto de la educación sexual es netamente biologicista, lo que ha determinado la forma en que se enseña, los imaginarios utilizados y la transmisión de mensajes con pretensiones de legitimidad (Bernstein, 1990; Galaz, Troncoso y Morrison, 2016).

De esta forma, más que educar en la igualdad, la salud y la responsabilidad (Cuellar, 2000), los programas de educación sexual "en su mayoría han puesto su acento en consideraciones biológico-reproductivas, prevención del embarazo, o exhortaciones morales que apelan al desarrollo del amor heterosexual" (Cornejo, 2014, p. 66). Morgade (2006) coincide con esta visión al señalar que la sexualidad en el ámbito educativo remite a la anatomía de la sexualidad, pero "desgajada de las emociones o de las relaciones humanas que le dan sentido al cuerpo biológico" (p. 42).

La consecuencia de este "tratamiento aséptico" de la diversidad sexual ha sido su invisibilización y el desconocimiento por parte de los miembros de las comunidades educativas (Suárez y Villegas, 2015), lo que puede ser una explicación de las situaciones de violencia que viven muchas veces estudiantes LGTBI dentro de los establecimientos y bullying homofóbico (Cornejo, 2014; UNESCO, 2015).

En resumen, hoy nos enfrentamos a una revitalización de la discusión respecto de la diversidad sexual a nivel de las políticas públicas y en especial en el contexto educativo, toda vez que el concepto se ha posicionado como un valor democrático que determina la forma en que los Estados se hacen cargo del tema (Galaz et al., 2018), sin embargo, no se puede desconocer que las orientaciones que cada establecimiento tenga respecto de la concepción de diversidad sexual pueden marcar diferencias significativas en torno a las ideas y actuaciones que llevan a cabo con sus comunidades. Esto puede ser más claro por ejemplo si se considera la orientación laica o religiosa que puedan tener. En efecto, la adscripción a algún tipo de religión por parte de los centros educativos podría tener consecuencias concretas en las concepciones que se tengan acerca de la diversidad sexual por parte de la comunidad y, en especial, en la legitimidad que se atribuya a las expresiones afectivas no heterosexuales en su interior. En ese mismo sentido, las políticas, las prácticas e incluso la cultura inclusiva podrían ser también determinadas por la pertenencia religiosa de los colegios.

En ese marco, la investigación que da origen a este artículo se propuso como objetivo identificar las representaciones sociales -concebidas como creencias y actuaciones- que tienen docentes y estudiantes acerca de la inclusión de las temáticas y estudiantes LGTBI en un centro educativo de educación secundaria de orientación católica y otro laico de la ciudad de Santiago.

\section{Revisión de la literatura}

El concepto de diversidad tiene múltiples acepciones que abarcan variadas dimensiones que aluden a una "tipificación social, económica, política, religiosa o educativa y que por tanto colocan a la diversidad y a la diferencia dentro de un sistema tomando como referencia a los otros sistemas sociales" (Ramos, 2012, p. 94). Al conjunto de categorías mencionadas se suma aquella que alude a las opciones sexuales de las personas, bajo las cuales se incluye por ejemplo las orientaciones del deseo y prácticas sexuales y también la 
reconstrucción de las identidades sexuales (Peixoto, 2012). En una línea similar García (2007) incorpora al concepto de diversidad sexual no solo la vivencia subjetiva de la sexualidad, sino también las dimensiones biológicas, psicológicas y sociales que la constituyen.

Sin embargo, el concepto de diversidad sexual debe ser también concebido de manera relacional, vale decir, como diferente de una supuesta comunidad de igualesheterosexuales y en especial en cuanto a la situación de asimetría respecto de una práctica hegemónica del grupo mayoritario (Galaz et al., 2016).

Como una forma de abordar las "diversidades" existentes "en la década de los noventa comenzó a utilizarse la sigla LGTBI, reemplazando a LGB, que anteriormente había reemplazado a "comunidad gay" (Mejía y Almanza, 2010, p. 79). En la actualidad el Ministerio de Educación de Chile (2017) reconoce y utiliza esta sigla en documentos oficiales, dando cuenta de una apertura hacia la diversidad de orientaciones sexuales e identidades de género presentes en nuestra sociedad.

Por su parte, con la incorporación de la idea de inclusión en el ámbito educativo también se han ampliado las concepciones tradicionales asociadas a las necesidades educativas especiales. Como señala Rojas y Armijo (2016), esta nueva perspectiva ha implicado que la inclusión en el espacio educativo supone el convivir con toda la gama de diversidades, dentro de la cual tiene plena cabida la diversidad sexual, teniendo siempre presente que la inclusión no solo hace referencia a personas que presentan alguna dificultad tanto física como psicológica, sino que también a toda barrera que limite el aprendizaje y la participación de los estudiantes, por tanto sería un proceso de desarrollo que no tiene fin ya que siempre existe la posibilidad de que se presenten nuevas barreras (Booth y Ainscow, 2000).

En este sentido, si bien la inclusión remite a un valor y una suerte de aspiración ética (Echeita, 2008), no se "puede desconocer que existen grupos que por sus características presentan mayores riesgos de vivir en forma efectiva ese ideal de inclusión” (Poblete y Galaz, 2017, p. 242).

Bajo esta lógica la escuela juega un rol clave en la construcción y reconstrucción del universo simbólico bajo el cual se forma la identidad de niños y niñas, en especial porque no es solo un espacio de aprendizajes o transmisión de conocimientos, sino también de convivencia entre diversos (Peixoto, 2012) que puede aspirar a generar una cultura de respeto y aceptación mutua.

De ahí entonces que los centros educativos debiesen visibilizar el tema de la diversidad sexual, siendo los profesores actores importantes por lo que "la identidad sexual se configura como otro de los conocimientos que deberían formar parte del conocimiento y saber pedagógico de un profesor o profesora que ejerce en tiempos actuales" (Julio et al., 2016, p. 55). Pero también deben ser los/as propios/as estudiantes quienes encarnen los valores democráticos que impliquen una convivencia basada en el respeto. De esta forma se concibe la acción de la escuela y de sus actores como clave porque "puede jugar un papel significativo en la erradicación del racismo y de otras formas de enfrentamiento interhumano (esto es la mentalidad del nosotros versus ellos), haciendo un esfuerzo consciente para exponer las bases de los mitos que rodean y justifican la superioridad de unos grupos humanos sobre otros" (García y Sáez, 1998, p. 31 ). 
La forma en que tradicionalmente se ha abordado el tema de la diversidad sexual en las escuelas ha sido a través de la educación sexual, entendida como "el intento de transmisión de las concepciones, normas y valores morales e ideología que cada cultura considera que deben servir para preservar el modelo social, cultural y económico" (Barragán, 1999, p.17). Al respecto, en los modelos utilizados en Chile ha primado una transmisión heteronormativa de la sexualidad, sujeta a marcos conservadores y una compresión biologicista de las relaciones interpersonales (Galaz et al., 2018).

En efecto, hablar de diversidad sexual en el marco de las perspectivas educativas tiene implicancias problemáticas dado que se reproduce una idea reducida de otredad (Barret, 1990), reificando la mayor parte de las veces un ideal heteronormativo de normalidad (Galaz et al., 2016).

A nivel de las políticas públicas, al menos desde el retorno a la democracia en Chile, el tratamiento de la diversidad sexual ha transitado por una visión restringida que enfatiza sus aspectos biológico-reproductivos incluso con apelaciones morales que reivindican el amor heterosexual (Cornejo, 2014).

En efecto, la mayor parte de los programas "fijan normas sobre información, orientación y prestaciones en materia de regulación de la fertilidad dejando en un plano secundario otros temas relacionados como las vivencias y expresiones de deseo" (Galaz et al., 2018, p. 4).

Como una variable significativa en torno al tratamiento del tema -y amparado en la libertad educativa que consagra la Ley Orgánica Constitucional de Educación- aparece el hecho que cada escuela puede escoger si aplica algún tipo de programa de educación sexual. Por tanto, serán los proyectos educativos los que definan la orientación que desea otorgarle. En esa línea, es claro que pueden existir diferencias significativas en el abordaje de estos temas al tratarse por ejemplo de un colegio de orientación católica u otro laico. E incluso las representaciones sociales del profesorado y estudiantes sobre la diversidad sexual y la inclusión pueden marcar comportamientos colectivos y actitudes hacia quienes encaran la diferencia.

Las evidencias señalan que por lo general son las creencias y representaciones sociales del profesorado las que pueden representar un riesgo para los procesos educativos que tienen como objetivo el respeto de la diversidad (López e Hinojosa, 2012). En ese sentido, entendemos las representaciones sociales como una expresión colectiva y una "forma de pensamiento que impera en una sociedad y que irradia a todos sus integrantes. El individuo se constituye en persona mediante la incorporación de este pensamiento colectivo, constituido por normas, valores, creencias, mitos" (Piña, 2004, p. 105), es decir, las representaciones colectivas son fruto de una comunidad en su conjunto y por tanto lo colectivo está por sobre lo individual. Liscano y Jurado (2016) afirman que las representaciones sociales suponen aspectos que componen la realidad física y social de los sujetos, por lo que determinan comportamientos y formas de actuación en un contexto determinado. En esa misma línea, Mora (2002) plantea que existen tres dimensiones de las representaciones sociales, la actitud, información y representación, las que en conjunto determinan las actuaciones de grupo o personas frente a determinadas situaciones o realidades.

Por lo mismo, las representaciones sociales del profesorado hacia la diversidad sexual no solo determinan el trato que reciben los y las estudiantes LGTBI, sino también "la 
posibilidad de aportar a una cultura inclusiva al interior de las escuelas, entendida como una comunidad que promueve valores coherentes con los principios de la democracia, solidaridad y el respeto irrestricto a la diversidad" (Cerón, Pérez y Poblete, 2017, p. 236).

\section{Método}

El objeto de estudio de esta investigación son las representaciones sociales, entendidas como actuaciones y creencias (Mora, 2002) del profesorado y estudiantes respecto de la inclusión educativa de estudiantes LGTBI en un liceo católico y otro laico. La investigación se llevó a cabo bajo un paradigma interpretativo (Gadamer, 1997) asumiendo que el significado que los sujetos otorgan a su realidad es clave para lograr un acercamiento profundo al objeto de estudio. Esta perspectiva de trabajo supone, además, la generación de una estrecha relación entre la realidad investigada y quien la investiga (Ruiz, 2012). Por lo mismo se priorizó un enfoque cualitativo debido a que en este tipo de indagación se pretende comprender los significados de las interacciones humanas a través del lenguaje (Ruiz, 2012), esto en concordancia con Canales (2006) quien establece que las investigaciones cualitativas se mueven en torno a la reproducción de la comunidad y su intento de análisis y comprensión.

Con una finalidad descriptiva, la investigación se llevó a cabo en dos liceos de la ciudad de Santiago de Chile, uno de las comunas de San Bernardo, particular subvencionado y católico, y otro de la comuna de Ñuñoa, de carácter público y laico. En cada caso se escogió una muestra intencionada (Alaminos, 2006) de estudiantes de educación secundaria y docentes de diferentes asignaturas de primero a cuarto medio. Para el caso de los/as estudiantes se tomaron en consideración criterios asociados a su permanencia en el establecimiento (mínimo un año); curso (primero a cuarto medio) y paridad de género, mientras que para el caso del profesorado especialidad, carga horaria (igual o superior a 20 horas) y años de experiencia en los establecimientos (mínimo dos). De esta forma, se trabajó con un total de 16 participantes ( 8 estudiantes y 8 docentes) a quienes se les aplicó una entrevista en profundidad individual y semiestructurada (Hernández, Fernández y Baptista, 2010).

El instrumento constó de 11 preguntas; 7 de ellas asociadas a las creencias y 4 a los comportamientos de profesores frente a la comunidad LGTBI y fue sometido a la validación de especialistas como una forma de asegurar su fiabilidad interna (Erazo, 2011). En forma adicional se consideraron como criterios de rigor científico la credibilidad, lograda a partir de la transcripción literal de los datos (Noreña et al., 2012) y su posterior análisis; consistencia, asumida desde la estabilidad de los datos (Erazo, 2011), toda vez que fueron obtenidos en el contexto propio de los y las participantes y sometidos a contrastación.

A partir de los datos recogidos se llevó a cabo un proceso de triangulación de teorías (Benavides y Gómez-Restrepo 2005), ya que se establecieron previamente categorías de análisis (actuaciones y creencias de docentes y estudiantes hacia la inclusión de estudiantes LGTBI), lo que permitió contrarrestar y corroborar las diferentes perspectivas de las interpretaciones realizadas a partir de las entrevistas, poniendo entonces a la triangulación como un procedimiento indispensable para enriquecer las representaciones (Flick, 2014; Pérez, 1998). 
Finalmente, se utilizó la técnica de análisis de discurso como herramienta para el trabajo con los datos provenientes de las entrevistas en atención a sus dimensiones características de uso del lenguaje, comunicación e interacción (Amezcua y Gálvez, 2002).

\section{Resultados}

\subsection{Creencias}

Las creencias de los diferentes actores que participaron en el estudio, tanto estudiantes como docentes, se mueven dentro de dos aspectos relevantes: el primero de ellos alude a la importancia de contar con una legislación e instrumentos normativos que regulen el acceso y permanencia de los/as estudiantes LGTBI en los liceos. En segundo lugar, el reconocimiento-invisibilización de quienes forman parte de la comunidad LGTBI en los establecimientos, lo que muchas veces se traduce en la incapacidad de reconocer al otro/a, abriendo la puerta a ciertos temores que expresan los/as estudiantes para explicitar su condición u orientación sexual abiertamente.

En relación con lo primero, desde el ámbito normativo se reconoce el valor de contar con una legislación a favor de las personas LGTBI como la recientemente aprobada ley de identidad de género, la ley de inclusión educativa y la ley Zamudio. Estos cuerpos legales contribuyen a la inclusión educativa de los estudiantes LGTBI y la no discriminación, toda vez que se valora la capacidad reguladora que tienen sobre el espacio educativo, instalando una lógica discursiva centrada en el valor de la aceptación que funciona como una suerte de resguardo en torno a la identidad: "Yo creo que es buena, creo que es necesaria (...) de cierta manera la ley los protege y los ayuda a ser ellos mismos" (Docente Liceo Católico).

Un punto relevante de este relato es lo que dice relación con la posibilidad de ser ellos mismos, lo que surge debido a que los cuerpos legales mencionados prohíben cualquier tipo de discriminación basada en las orientaciones sexuales de las personas. Sin embargo, aunque esto es positivo, preocupa el desconocimiento que persiste respecto de estas normas en los/as estudiantes y la crítica que deslizan hacia sus establecimientos en relación a la ausencia de políticas efectivas que hagan "aplicables" las leyes mencionadas.

\section{Está bien, pero primero que nada como que según yo no debería existir porque debería ser algo intrínseco que cada que establecimiento los incluya, pero como eso no ocurre es muy utópico, está bien que se cree una ley para influenciar como el, como se dice, la unión de todos los sectores sexuales. (Estudiante Liceo Católico)}

De este relato se desprende que se reconoce el valor de la ley, pero por sobre todo que sean los propios establecimientos los que incluyan y no generen discriminaciones sin necesidad de una ley que los obligue. Esta idea es común tanto a los/as estudiantes del liceo católico como laico, toda vez que no existen diferencias en este tipo de apreciación, situando una responsabilidad en la institucionalidad y en especial en el mundo adulto. Al respecto, la legitimidad de las normas está dada por un valor de lo "políticamente correcto", sin embargo, son las propias instituciones educativas las que debieran generar mecanismos que favorezcan la inclusión y no discriminación en especial para los grupos LGTBI, más si se considera el caso del liceo católico donde el conjunto de entrevistados (profesores/as y estudiantes) señala que no existen estudiantes LGTBI. De acuerdo a Goffman (2006), ocurre que muchas veces los/as estudiantes de grupos minoritarios realizarían esfuerzos por ocultar su identidad, destacándose en otros ámbitos, de tal manera que el estigma que pueda rondar en torno a ellos no sea lo que se prime, 
escondiendo y encubriendo así su verdadera identidad, como por ejemplo, lo plantea este estudiante: "en esta comunidad, yo no he sabido de alguien que sea un estudiante LGTBI, pero hay personas como que dan la impresión, por ejemplo el mismo presidente del centro de alumnos" (Estudiante Liceo Católico)...”yo creo que ha habido, uno tiene sospechas de algunos niños" (Docente Liceo Católico).

En ambos casos aparece una atribución o sospecha de alguien que podría ser. Sin embargo, es preferible ocultarlo: "que uno no sepa si se dan o no estas relaciones es porque se tapa, porque de saberlo ¿̇qué pasaría con ese estudiante?”. (Docente Liceo Católico)

La pregunta final del relato citado pone en evidencia una suerte de temor respecto de lo que ocurriría si un/a estudiante declara su orientación no heterosexual abiertamente, asumiendo que se trataría de algo que escapa a lo normal, a lo culturalmente apto (Buttler, 2007). De acuerdo a lo indicado entonces por este docente, es preferible que persista el ocultamiento porque de revelarse se abre un espacio no conocido dentro del cual existe como posibilidad la discriminación, marginación y la estigmatización. De ahí que en muchos casos es preferible ocultar la propia identidad por temor (Goffman, 2006).

De hecho, la misma percepción la tienen los/as estudiantes, toda vez que reconocen que asumir públicamente una identidad no heteronormativa al interior del liceo puede traer problemas: "Si lo dice se arriesga mucho a la discriminación de la gente. Claro yo creo que sería apartado" (Estudiante Liceo Católico). "Ese alumno la pasaría mal porque no estamos preparados, no se nos enseña, no se nos habla del tema, se omite" (Estudiante Liceo Católico).

Lo interesante de ambos relatos es que se trata de un reconocimiento explícito acerca de la ausencia de un clima adecuado en el liceo para hablar estos temas abiertamente, porque "no estamos preparados". En rigor, se asume que esa falta de preparación podría generar situaciones de discriminación. Sin embargo, el llamado de atención es a la propia institución, dado que los problemas indicados derivan de la incapacidad de abordar el tema como comunidad, condenando al ocultamiento a quienes forman parte de la comunidad LGTBI.

Una situación diferente ocurre en el liceo laico, donde la comunidad LGTBI es identificada por estudiantes y profesorado como tal, sin que esto signifique temores a la marginación o rechazo.

En efecto, en este caso se trata de asumir que la diversidad sexual es una posibilidad que debe ser vivida con naturalidad y dentro de la cual se pueden generar relaciones abiertas y transparentes entre los estudiantes al margen de su orientación o condición sexual:

$$
\begin{aligned}
& \text { Yo considero que existe una buena relación, a pesar de todo lo anterior, considero se } \\
& \text { ha hecho un buen trabajo en relación a la inclusión en todos los ámbitos porque uno a } \\
& \text { veces cuando habla de inclusión solo se imagina las NEE y se imagina al niño en silla } \\
& \text { de ruedas, y acá en todo ámbito la inclusión, multicultural, LGBTI, NEE considero que } \\
& \text { se abarca súper bien tanto estudiantes y los profesores (...)los estudiantes yo encuentro } \\
& \text { que ellos aceptan, son respetuosos unos con los otros, jamás nosotros hemos tenido un } \\
& \text { caso de bullying por ejemplo con un alumno que se haya pintado las uñas o que ande } \\
& \text { con el pelo más largo. (Docente Liceo Laico) }
\end{aligned}
$$

En la misma línea:

To soy bisexual y nada, yo, yo venía de un colegio distinto, ese colegio es como más liberal que acá y yo me estaba asustando porque era como ah ok, lo van a tomar mal, con mis amigas una vez lo dije y lo dije como normal porque se supone que es normal, 
pero yo pensé que me iban a decir algo y fue como o wow, pero en si no discriminan así, hay varios también que son gays, creo que hay un par de chicos que son trans, así que no hay ningún tipo de discriminación por lo menos yo no lo he visto así. (Estudiante Liceo Laico)

Claramente, ambos relatos coinciden en vivir con cierta naturalidad la expresión abierta de las orientaciones sexuales de los y las estudiantes al interior del liceo. De hecho, se asume la idea que no es necesario ocultarse, como si ocurría en el liceo católico. Concretamente, uno de los docentes entrevistados señala:

\section{No para nada, ellos tienen que vivir como consideren, como ellos se aceptan y ellos se quieren, y si el día de mañana un hombre decide ser una mujer y si él se acepta y se ama así, todos los tenemos que aceptar porque él lo decidió así y es su vida y si Juanito el día de mañana decide ser maría que sea feliz. (Docente Liceo Laico)}

Pareciera, entonces, que la disposición institucional a trabajar estos temas favorece la instalación de un clima adecuado. Se trata de reconocer en la variable política un factor clave en la promoción de relaciones respetuosas de la diversidad y en la inclusión de todos y todas (Booth y Ainscow, 2000).

Pese a esta apertura que se da al interior de este establecimiento y las positivas perspectivas que se observan entre estudiantes y docentes en torno al tema, no se puede desconocer que existen riesgos fuera del espacio educativo, especialmente en una sociedad en la cual persisten conductas discriminatorias hacia las personas LGTBI:

\footnotetext{
Riesgo de la sociedad, como que los vaya a rechazar, en ese aspecto siempre va a haber alguno que no esté de acuerdo, siempre va a existir gente que los va a humillar, que los va a violentar física o verbalmente, pero esas situaciones van a depender de ellos...pero en si estos temas se han abierto tanto que las personas de a poco han comenzado a aceptar, lamentablemente muchas veces la sociedad se transforma en un enemigo. Entonces creo que hay riesgos, pero se pueden solucionar. (Docente Liceo Laico)
}

La constatación de estos riesgos es común a todos los actores (estudiantes y docentes) y en ambos establecimientos, dado que se reconoce la presencia de una heteronormatividad que muchas veces da paso a discriminaciones y bullying homofóbico (Cornejo, 2014; UNESCO, 2015).

\section{La presencia de esta idea puede explicar una visión en torno a lo que es normal y lo que no lo es. En efecto, en el liceo católico en algunos entrevistados se pudo constatar que bajo sus criterios la comunidad LGTBI estaría fuera de la norma: "si están ocultando algo ya no son normales, si no son normales hay una parte que no está funcionando bien". (Profesor Liceo Católico)}

Claramente hay una patologización de la orientación no heterosexual, con lo cual se deja en evidencia una visión de escuela normalizadora que debe promover una identidad uniforme y homogeneizante (Poblete, 2009). Por lo mismo, es necesario dejar de hacer una distinción entre normales y no normales, y pasar a una mirada inclusiva en donde se consideren y valoren las características individuales de cada integrante de la comunidad educativa.

\subsection{Actuaciones}

Si bien las creencias o percepciones que se tengan respecto de la inclusión de determinados grupos en el espacio educativo son claves (Jordán, 1994; Ortiz, 2008; Cerón et al., 2017), las acciones también juegan un rol determinante, dado que estas tienen una capacidad de incidencia que se traduce muchas veces en los mecanismos políticos o procedimentales que 
se pueden implementar para alcanzar los valores y prácticas fundados en los derechos humanos que supone la inclusión (Blanco, 1999; Booth y Ainscow, 2000; Echeita, 2008).

En esa línea, entre los principales hallazgos que surgen de la investigación, aparece el hecho que los temas LGTBI no son trabajados ni tampoco se promueven acciones específicas de forma institucional para favorecer su inclusión, en especial en el liceo católico. Si bien se realizan actividades que tienen por objetivo el respeto hacia las personas en general, no se visibiliza el tema LGTBI como un grupo de interés particular. Esto es coherente con lo señalado por Ramos (2012), al indicar que cada comunidad construye su propio significado de diversidad y que en este caso particular no considera aquella que deriva de la diversidad sexual.

El punto es que bajo la idea de diversidad se busca subsumir a todos aquellos grupos que responden a conductas no hegemónicas, funcionando como una especie de término "sombrilla" que admite múltiples alternativas, pero que invisibiliza las diferencias y particularidades. En efecto, la utilización de la diversidad bajo esta mirada amplia, pero restrictiva a la vez, no permite ver la construcción de subjetividades o los procesos de dominación y resistencia (Foucault, 1977) que muchas veces se suceden en el ámbito escolar.

Respecto de esta invisibilización del tema, un docente señala:

No, aquí no hacemos nada, no es que yo creo que aquí en el colegio es como que no existe este tema, es como una cosa que nosotros nos hacemos los lesos, y ni siquiera es que no lo aceptamos, si no, que es como que no existe, entonces menos podemos sentarnos a conversar o hacer proyectos y programas de nada ese problema, no hay posibilidades.

(Docente Liceo Católico)

De acuerdo a lo expresado, queda claro que no está dentro de las prioridades institucionales llevar a cabo acciones educativas con su comunidad en torno al tema. Sin embargo, aunque la opción institucional es la invisibilización, algunos profesores realizan acciones de forma individual:

Yo hablo abiertamente con los chiquillos, aunque sé que va en contra de la institución
a la que represento, pero les digo que eso es lo que yo pienso, que es mi opinión personal
y lo hago porque los estudiantes no pueden salir de aquí pensando que el mundo sólo
es blanco y negro, por lo que es necesario que conozcan acerca de las diferentes
realidades que existen y de lo contrario pueden actuar maly causar daño. (Profesora
Liceo Católico)

Una situación diferente se vive en el liceo laico en donde las actuaciones de profesores y estudiantes dan cuenta de cómo paulatinamente se han hecho cargo del tema de la diversidad sexual. Incluso se menciona un caso de interés que involucró a la comunidad: "Yo conozco a una chica que ahora pasó a ser chico, que intentó realizarse el tratamiento de hormonas y le salía muy caro, por eso pidió ayuda a todos y la comunidad en general lo hizo” (Estudiante Liceo Laico).

Sin embargo, más allá de estas acciones puntuales, también hay una política institucional respecto del tema, tal como manifiesta este profesor: "Este año nosotros aplicamos una unidad de diversidad sexual que tuvo una muy buena llegada y que hablaba de estos temas" (Profesor Liceo Laico).

Se trata, entonces, de una voluntad explícita de parte de docentes y directivos de hacer que la diversidad sexual se discuta abiertamente en el espacio formativo, de forma tal que en determinadas asignaturas se aborden estos temas, como por ejemplo en las horas 
destinadas a orientación. Ahora bien, es relevante destacar que esta omisión no siempre se debe a problemas de "voluntad", sino también al desconocimiento que existe sobre las implicancias de la diversidad sexual y sus expresiones, lo que aparece como un problema compartido en ambos establecimientos.

Esto es coincidente con lo señalado por el Movilh (2008) que sostiene que la falta de conocimiento es una limitante a la hora de generar actividades inclusivas, lo que se expresa en que muchos/as docentes declaran no sentirse preparados para enfrentar la homosexualidad y/o transexualidad en sus centros educativos ni mucho menos en sus aulas.

De ahí la importancia de generar acciones de formación y capacitación e incluso promover cambios en la formación inicial docente para fortalecer las prácticas inclusivas dentro de los establecimientos educacionales. Esto es de vital importancia puesto que, a la larga, tal como plantea Julio y colaboradores (2016), los profesores pueden llegar a transformarse en obstaculizadores del proceso de construcción de la identidad sexual de sus estudiantes.

\section{Conclusión}

La preocupación por la inclusión educativa y el valor de la diversidad, su reconocimiento y respeto, se ha posicionado como un principio presente en las normas y políticas públicas en Chile. En efecto, no solo la reciente legislación se hace cargo de estos principios, sino también las políticas que por ejemplo el Ministerio de Educación ha ido elaborando. Sin embargo, lo que ocurre con la diversidad sexual no ha logrado el mismo nivel de posicionamiento. En parte porque la construcción discursiva que se hace de esta "otredad" es realizada en relación a un sistema de privilegio y una matriz heterosexual (Galaz, et al. 2018).

Esto mismo tiene un reflejo en la forma en que se ha ido abordando la educación sexual en el país, dado que no existe a la fecha una política que se sitúe desde una perspectiva no normativa. La pregunta que habría que hacerse, siguiendo a Morgade (2011), es cuánto de la forma en que se abordan estos temas finalmente tiene por objetivo preservar el orden de género establecido.

Esta misma carencia se observa en los establecimientos educacionales, en tanto persisten creencias y actuaciones que restringen las concepciones en torno a la diversidad sexual y los grupos LGTBI. Incluso los y las docentes que participaron de este estudio indican no sentirse preparados para abordar las situaciones que derivan de la presencia en sus centros educativos y aulas de estudiantes LGTBI.

Sin embargo, estas dificultades tienden a agudizarse cuando se hace la distinción respecto de la orientación de cada uno de los liceos estudiados. En efecto, en el liceo católico se trata de un tema que se invisibiliza bajo el pretexto de resguardar la integridad de sus estudiantes y por temor a lo que ocurriría si alguien asume abiertamente su identidad sexual no heteronormativa. Mientras, en el liceo laico, existe una mayor apertura de parte del profesorado y sus estudiantes para hacerse cargo de las situaciones que derivan de la presencia de estudiantes LGTBI, las que no se conciben como problemáticas. De ahí que las actuaciones o prácticas que se llevan a cabo permiten que por ejemplo en determinadas asignaturas se hable abiertamente de estos temas, lo que no ocurre en el liceo confesional. De esta forma, las comprensiones respecto de lo que significa la inclusión aparecen como restringidas en este último establecimiento, porque sus fundamentos están más en la 
normalización de los y las estudiantes, que en la construcción de una otredad diferente (Matus y Rojas, 2015) que surge precisamente del reconocimiento de ésta.

La modificación de las creencias y en especial de las prácticas en orden a lograr la inclusión de la diversidad sexual en el ámbito educativo es un proceso que lleva tiempo, más si se asume que su fundamento está en el cuestionamiento de la matriz socio-histórica de la dominación (Ocampo, 2016), que ha justificado exclusiones explícitas de determinados grupos sociales. Por lo mismo, la reflexión en torno a los factores que favorecen la inclusión en los centros educativos es cada vez más relevante, en especial cuando se asocia a grupos y personas que no se inscriben en las lógicas normativas dominantes.

Los derechos humanos de todos y todas no puede ser solo un ideal, por lo cual la modificación de las estructuras tradicionales en los espacios educativos es clave para avanzar en su concreción. Al respecto, la revisión de las culturas, políticas y prácticas ofrece un conjunto de opciones y desafíos para avanzar hacia la inclusión, sin embargo, las propias representaciones y creencias de los actores sociales son muchas veces los principales obstáculos que se requiere vencer. De ahí la importancia de generar conocimientos en torno a estas temáticas y lograr sociedades más justas y democráticas, en cuya construcción el sistema educativo juega un rol central.

\section{Referencias}

Alaminos, A. (2006). El muestreo en la investigación social. En A. Alaminos, y J. L. Castejón (Eds.), Elaboración, análisis e interpretación de encuestas, cuestionarios y escalas de opinión (pp. 41-67). Alicante: Marfil.

Amezcua, M. y Gálvez, A. (2002). Los modos de análisis en investigación cualitativa en salud: Perspectiva crítica y reflexiones en voz alta. Revista Española de Salud Pública, 76(5), 423436. https://doi.org/10.1590/S1 135-57272002000500005

Arenas, L., Duran, J., Dides, C. y Fernández C. (2016). Primer informe salud sexual salud reproductiva y derechos humanos. Santiago de Chile: Corporación Miles.

Astudillo, P. (2016). La inestable aceptación de la homosexualidad. El caso de las escuelas católicas de élite en Santiago de Chile. Revista Latinoamericana de Educación Inclusiva, 10(2), 21-37. https://doi.org/10.4067/So7 18-73782016000200003

Barbero, M. y Pichardo, J. (2016). Sumando libertades. Guía iberoamericana para el abordaje del acoso escolar por homofobia y transfobia. Bogotá: Red Iberoamericana de Educación LGBTI.

Barragán, F. (1999). Programa de educación afectivo sexual. sexualidad, educación sexual y género. Sevilla: Junta de Andalucía.

Barret, M. (1990). El concepto de diferencia. Debate Feminista, 1, 311-325. https://doi.org/10.22201/cieg.2594066xe.1990.2.1931

Benavides, M. y Gómez-Restrepo, C. (2005). Métodos en investigación cualitativa: Triangulación. Revista Colombiana de Psiquiatría, 34(1), 118-124.

Bernstein, B. (1990). Poder, educación y conciencia. Sociología de la transmisión cultural. Barcelona: El Roure.

Blanco, R. (1999). Proyecto principal de educación para América Latina y el Caribe. Boletín, 48, 5572.

Booth, T. y Ainscow, M. (2000). Guía para la evaluación y mejora de la ecuación inclusiva. Index for inclusion. Madrid: Consorcio Universitario para la Educación Inclusiva. 
Buttler, J. (2007). El género en disputa. El feminismo y la subversión de la identidad. Barcelona: Paidós.

Canales, M. (2006). Metodologías de investigación social. Santiago de Chile: LOM.

Cerón, L., Pérez, M. y Poblete, R. (2017). Percepciones docentes en torno a la presencia de niños y niñas en escuela de Santiago: Retos y desafíos para la inclusión. Revista Latinoamericana de Educación Inclusiva, 1 1(2), 233-246. https://doi.org/10.4067/So718-73782017000200015

Cornejo, J. (2014). Bullying homofóbico en Chile: Trayectoria histórica. Límite, 9(30), 61-70.

Cornejo, J. (2018). Discriminación y violencia homofóbica en el sistema escolar: Estrategias de prevención, manejo y combate. Revista Brasileira de Educação, 23, e230031.

https://doi.org/10.1590/s1413-24782018230031

Echeita, G. (2008). Inclusión y exclusión educativa. Voz y quebranto. REICE. Revista Iberoamericana sobre Calidad, Eficacia y Cambio en Educación, 6(2), 9-18.

Erazo, M. (2011). Rigor científico en las prácticas de investigación cualitativa. Ciencia, Docencia y Tecnología, 21(42), 107-136.

Flick, L. (2014). An introduction to cualitative research. Nueva York, NY: SAGE Publications.

Foucault, M. (1977). Vigilar y castigar. Madrid: Siglo XXI.

Gadamer, H. (1997). Verdad y método. Madrid: Sígueme

Galaz, C., Troncoso, L. y Morrison, R. (2016). Miradas críticas sobre la intervención educativa en diversidad sexual. Revista Latinoamericana de Inclusión Educativa, 10(2), 93-111. https://doi.org/10.4067/So7 18-73782016000200007

Galaz Valderrama, C., Sepúlveda Galeas, M, Poblete Melis, R., Troncoso Pérez, L. y Morrison Jara, R. (2018). Derechos LGTBI en Chile: Tensiones en la constitución de otredades sexualizadas. Psicoperspectivas, 17(1), 24-47. https://doi.org/10.5027/psicoperspectivasVol17-Issue 1-fulltext-1 165

García, A. Sáez, J. (1998). Del racismo a la interculturalidad. Madrid: Narcea

García, C. (2007). Diversidad sexual en la escuela, dinámicas pedagógicas para enfrentar la homofobia. Bogotá: Colombia Diversa.

Goffman, E. (2006). Estigma. La identidad deteriorada. Madrid: Amorrortu.

González, M., Núñez, C. G., Galaz Valderrama, C., Troncoso, L. y Morrison Jara, R. (2018). Los usos de la diversidad sexual en la acción pública. Psicoperspectivas, 17(1), 17-35.

https://doi.org/10.5027/psicoperspectivas-Vol17-Issue1-fulltext-1 165

Hernández, R., Fernández, C. y Baptista, P. (2010). Metodología de la investigación. Ciudad de México: McGraw-Hill.

Jordán, J. (1994). La escuela multicultural: Un reto para el profesorado. Barcelona: Paidós.

Julio, C. Kaeufer, A., Riquelme, C., Silva, M., Osorio, M. y Torres, N. (2016) Conocimientos sobre identidad sexual de profesores y profesoras: ¿Barreras y facilitadores de construcción identitaria? Revista latinoamericana de Educación Inclusiva, 1O(2), 53-71. https://doi.org/10.4067/So7 18-73782016000200005

Liscano, D. y Jurado, P. (2016). Representaciones sociales sobre las personas LGBTI en la universidad: Perspectivas del profesorado y alumnado. Revista Nacional e Internacional de Educación Inclusiva, 9(3), 231-249.

López, M. e Hinojosa, E. (2012). El estudio de las creencias sobre la diversidad cultural como referente para la mejora de la formación docente. Educación XXI, 15(1), 195-218.

https://doi.org/10.5944/educxx 1.15.1.156 
Mejia, J. y Almanza, M. (2010). Comunidad LGBT: Historia y reconocimientos jurídicos. Revista Justicia, 17, 78-110.

Ministerio de Educación de Chile. (2017). Orientaciones para la inclusión de las personas lesbianas, gays, bisexuales, trans e intersex. Santiago de Chile: Chile.

Monzón, C. (2017). "Bus de la libertad" versus "bus de la diversidad": Así fue el esperado cara a cara. Santiago de Chile: Publimetro.

Mora, M. (2002). La teoría de las representaciones sociales de Serge Moscovici. Revista Athenea Digital, 2, 1-25. https://doi.org/10.5565/rev/athenea.55

Morgade, G. (2006). Educación en la sexualidad desde el enfoque de género. Una antigua deuda de la escuela. Novedades Educativas, 184, 40-44.

Morgade, G. (2011). Toda educación es sexual: Hacia una educación sexuada justa. Buenos Aires: La Crujía Ediciones.

Noreña, A., Alcaraz, N., Rojas, J. y Rebolledo, D. (2012). Aplicabilidad de los criterios de rigor y éticos en la investigación cualitativa. Aquichan, $12(3), 263-274$. https://doi.org/10.5294/aqui.2012.12.3.5

Núñez, L. (2014). Autora de "Nicolás tiene dos papás: El cuento “no expresa ninguna ideología”. Santiago: Emol.

Ocampo, A. (2015). Aproximaciones y descripciones generales sobre la formación del objeto de la educación inclusiva. En A. Ocampo (Coord.), Los rumbos de la educación inclusiva en los inicios del siglo XXI: Cartografias para modernizar el enfoque (pp. 24-90). Santiago de Chile: CELEI.

Ortiz, M. (2008). Inmigración en las aulas: Percepciones prejuiciosas de los docentes. Revista de Sociología Papers, 87, 253-268. https://doi.org/10.5565/rev/papers/v87no.805

Peixoto. (2012). Escuela y diversidad sexual- ¿Qué realidad? Educacao em Revista, 28(3), 143-158. https://doi.org/10.1590/S0102-46982012000300007

Piña, J. (2004). La teoría de las representaciones sociales. Su uso en la investigación educativa en México. Perfiles Educativos, 26(105-106), 102-124.

Poblete, R. (2009). Educación intercultural en la escuela de hoy: Reformas y desafíos para su implementación. Revista Latinoamericana de Educación Inclusiva, 3(2), 181-200.

Poblete, R. y Galaz, C. (2017). Aperturas y cierres para la inclusión de niños/as migrantes en Chile. Revista Estudios Pedagógicos, 43(3), 239-257. https://doi.org/10.4067/So7 18-07052017000300014

Ramos, J. (2012). ¿Cuándo se habla de diversidad de que se habla? Revista Iberoamericana de Educación de Adultos, 34(1), 76-96.

Rehbein, C. (2017). 61\% de escolares acusa bullying hacia el alumnado LGBTI por parte de los docentes. Santiago de Chile: Publimetro.

Ruiz, J. (2012). Metodología de la investigación cualitativa. Bilbao: Universidad de Deusto.

Suárez, L. y Villegas, T. (2015). Las opiniones docentes respecto de la diversidad de comportamiento de género y sus manifestaciones en el aula de clase grado cuarto de la institución educativa Byron Gavira (Trabajo fin de grado). Universidad Tecnológica de Pereira, Colombia.

Torres, J. (2013). Análisis del régimen jurídico de los manuales de convivencia en los colegios en relación con la discriminación de estudiantes LGBTI. Folios de Humanidades y Pedagogía, 1, 99-113.

UNESCO. (2015). La violencia homofóbica y transfóbica en el ámbito escolar: hacia centros educativos inclusivos y seguros en América Latina. Santiago de Chile: UNESCO. 


\section{Breve CV de los autores}

\section{Evelyn Toro Villagra}

Magister en Educación Inclusiva por la Universidad Central de Chile (2018). Licenciada en Educación y Profesora de física por la Pontificia Universidad Católica de Valparaíso, Chile (2012). Postítulo en educación con mención en matemáticas por la Universidad Central de Chile (2014). Ha desarrollado la mayor parte de su vida profesional como profesora de física en el Colegio Inmaculada Concepción donde fue encargada del Departamento de Ciencias y administradora pedagógica de recursos informáticos. Además, ha participado como ayudante de cátedra, laboratorio e investigación en diferentes proyectos de la Pontificia Universidad Católica de Valparaíso. ORCID ID: https://orcid.org/0000-0002-9225-5283. Email: evelyntoro.fisica@gmail.com

\section{Pablo Ignacio Moya}

Magister en Educación Inclusiva por la Universidad Central de Chile (2018). Licenciado en Educación y Profesor de Historia y Geografía por la Universidad San Sebastián, Chile (2016). Ha desarrollado la mayor parte de su vida profesional en el Archivo Nacional Histórico de Chile donde fue encargado del Departamento de Educación y vinculación con el medio; Además ha participado como ayudante de catedra e investigación en diferentes proyectos. ORCID ID: https://orcid.org/0000-0002-6051-3305. Email: pablomoyarojas@gmail.com

\section{Rolando Poblete Melis}

Doctor en Antropología Social y Cultural y Master en Investigación de la Universidad Autónoma de Barcelona, Licenciado en Filosofía por la Universidad Católica de Valparaíso. Es académico de la Facultad de Educación de la Universidad Bernardo O`Higgins e investigador del Centro de Investigación en Educación de la misma universidad. Integrante del núcleo interuniversitario Estudios Críticos de la Diversidad, sus áreas de trabajo son políticas públicas, migraciones, diversidad, género y educación. Sus últimas investigaciones y publicaciones están asociadas a los procesos de inclusión de niños y niñas migrantes en escuelas y en especial al trabajo con la diversidad que llevan a cabo los centros educativos. ORCID ID: https://orcid.org/0000-0002-7799-7190. Email: rolando.poblete.melis@gmail.com 